\title{
Creating virtual communities of practice for learning technology in higher education: issues, challenges and experiences
}

\author{
Jacqueline A. Dempster," Helen Beetham,** Peter Jackson* and \\ Steven Richardson***** \\ *University of Warwick, *光University of Bristol, ***University of Manchester \\ email:jay,dempster@wanwickac.uk
}

The need for a Web portal to support the rapidly growing field of learning technology has been well established through a number of national surveys and scoping studies over recent years. The overarching vision has been the provision of a virtual environment to assist in informing and developing professional practice in the use of learning technologies. This paper outlines the issues and challenges in creating such a portal through the experiences of developing the RESULTS Network. In the paper, design and participation issues are considered within the wider context of online and networked approaches to supporting practice and professional development. User participation methodologies and technical developments for RESULTS are described in relation to a review of existing representations of practice and a comprehensive survey amongst the learning technology users' community. An outline of key achievements and experiences is presented, followed by some conclusions regarding the cultural and political issues in creating a viable and sustainable facility and suggestions for possible future direction in national provision.

\section{Introduction}

Most higher education institutions have taken seriously the recommendations in the Dearing Report concerning greater use of technology for learning and teaching (National Committee of Inquiry into Higher Education (NCIHE), 1997). Each year, more teaching staff are making use of Information and Communication Technology (ICT) tools and environments with their students, or integrating digital resources into their courses. It is increasingly evident (Armitage, Rothery and Jenkins, 1999; Beetham, 2000; Beetham, Jones and Gornall, 2001) that nearly all universities and colleges now provide some form of specific support for learning technology, indicated in the number of new posts and support units created for this area. The result is a rapidly growing group of multi-skilled 
professionals, employed both within institutions and/or nationally funded learning technology programmes and services, which have been such a key feature of the United Kingdom experience.

As well as 'learning technologists', other categories of staff, such as librarians and IT developers, teaching support staff, educational developers and institutional managers, are developing new capabilities and understanding in learning technology (Gornall, 1999; Gibbs, 2000; Beetham et al., 2001). Many such staff also have responsibility for brokerage of information and networking (Oliver, 2002).

It is notable that in a growing number of institutions and in national programmes, a major theme appears to be to capture lessons learned on the use of ICT and, in particular, to improve the dissemination and transfer of good practice. The abundance of new academic conferences and journals for learning technology is testimony to the high value placed on communication with others working in the field. However, learning technology roles and practices are diverse and poorly differentiated from one another and both the professional practice and the academic field of knowledge remain emergent (Conole, 2003).

\section{Supporting a learning technology community of practice}

A wide range of strategies is being used to support learning technology practice, including national support agencies, institutional initiatives and via professional development programmes (see review by Lally and McConnell, undated). There remains a feeling, however, that learning technology support staff, and the academics they work with, do not . have easy online access to high-quality information and resources which, thanks to the JISC and other initiatives, are now available. A number of national studies (notably TALiSMAN, 1999; Armitage et al., 1999; Gibbs, 2000) have identified significant gaps in provision of resources to assist staff involved with promoting and supporting the use of ICT in teaching and learning. Access to information and resources is felt to be patchy and finding materials is serendipitous and time-consuming.

Those working in the field of learning technology benefit enormously from collaboration and exchange of expertise with colleagues in similar roles in other institutions. It was therefore believed that the creation of a national portal to facilitate sharing and discussion of materials and methods used in leaming technology practice would be beneficial. This requires interoperability across the relevant professional and subject networks, but this takes several forms - technical, semantic, cultural, educational and political, and any portal developed would need to address this.

While this paper focuses on users of learning technologies, most of the factors, debates and outcomes overlap with other disciplines and are equally applicable to any educational portal supporting diverse communities of practice.

\section{Creating an online resource exchange and support network}

The RESULTs Network project was devised by Jay Dempster and Clive Young under the JISC-funded $5 / 99$ programme to provide a learning technology portal for United Kingdom higher education. The portal aimed to offer a focus for professional exchange and discussion and to fill the gap in information, resources and communication identified by 
the sector. RESULTs is the portal (www:results.ac.uk) and the RESULTs Network its wider community of users.

Providing a portal service that will support and help transform practice must be subject to an understanding and appreciation of the variety of identities and roles that practitioners take on, and the ways in which professional learning and teaching practice is changing. The research underpinning much of the theoretical and overview materials was commissioned early on and carried out by Beetham. Many of the key foundations of RESULTs stems from the findings of this user needs study (Beetham, 2001a) which included a literature review, an online survey, structured interviews and focus groups. The research instruments, survey methodology and study findings are described in detail in the full report (Beetham, 2001a).

The study set out to identify the potential users of a learning technology portal, the nature of their practice, and the kinds of resource and service that can support them in developing their practice effectively. Factors motivating participation were explored in depth. The research findings strongly indicated that the users wanted a community-orientated approach to contribution and annotation of resources. RESULTs was built on the vision of a rich information environment for user-defined resource submission, access and discovery coupled with facilities that encourage exchange and networking amongst communities of learning technology practice.

The project overall was led by Jay Dempster, and essentially has meant the development of a database-driven Web architecture, undertaken by Steven Richardson using specifications identified from the user participation studies and drawn up by Peter Jackson.

The RESULTs portal enables users to link to, categorize (and interlink) resources on the Web, directly integrated with discussion areas, as well as providing personalization features and user profiling. No such facility previously existed in any single and coherent sense. The portal aimed to tackle the multidisciplinary nature of learning technology. It enables and encourages cross-fertilization across diverse categories of users in the sector and supports a community approach to categorization and, as such, organic development of a consensusdriven taxonomy for learning technology.

\section{Representations of practice for a virtual community}

The theoretical and methodological touchstones for the user needs study and in turn for the RESULTs Network development arise from a number of existing educational frameworks for developing virtual communities of practice (Beetham, 2002). Lave and Wenger (1991) are the authors most closely identified with the idea of 'community of practice', which they describe as 'a set of relationships among persons, activity and world, over time, and in relation with other tangential and overlapping communities of practice'. It is evident from experiences of knowledge modelling (Stefik, 1995) that procedural and tacit knowledge of practice and expertise can be difficult to capture and are strongly biased towards subject knowledge and traditional modes of pedagogical practice in particular disciplines. The ASTER project, for example, has found that terms such as 'seminar' or 'tutorial' are open to interpretation and can relate to completely different sets of pedagogical practices in different subject areas (ASTER, 1999). 
In analysing online professional development networks, Foster, Bowskill, Lally and McConnell (2000) found that they have several prerequisites: facilities for asynchronous discussion, shared information resources, tools (for collaboration itself, and to support aspects of off-line practice), and investment of time and resource, both from the 'centre' and from participants. Action learning (Beaty and McGill, 1995) is likely to be a factor in motivating participation in such networks, since all members will be novices in some aspects of learning technology work but experts in others, and all will benefit from peer support in dealing with the 'real problems' of their practice.

The idea of organizing a professional network around specific shared activities brings in another series of possible models from the literature on computer-supported collaborative (or cooperative) work (CSCW: for example McConnell, 2000; Kollock and Smith, 2000; Baecker, 1992) and the investigation of groupware systems. CSCW is usually seen as involving two interlinked activities: peer-to-peer communication and the development of shared artefacts. The Virtual Learning Space (http:/lwww.vls.scotcit.ac.uk), a Scottish universities service, has used frequent real-time chat sessions and access to subject experts as a successful drive for participation. Another example is the Virtual Teacher Centre (http://vtc.ngfl.gov.uk), a professional practice community, which includes a Teacher Resource Exchange (http://contribute.ngfl.gov.uk) designed to help teachers develop and share ideas for activities in the classroom.

Finally, online professional development networks will have some features of an academic research community or subject portal as learning technologies become an object of academic study in their own right. Examples of good practice in United Kingdom higher education include SOSIG (http://www:sosig.ac. $u k$ ) and the Learning and Teaching Support Network (LTSN) Engineering portal (http://www:ltsneng.ac.uk). SOSIG offers a one-stop gateway to scholarly resources for students and academics in the social sciences. SOSIG uses 'Harvester' technology to automatically gather new resources from the Web, based on URLs from the existing SOSIG catalogue. The LTSN Engineering portal exists to enhance learning and teaching practice and is based not around content resources but orientated far more towards engagement with their end users.

\section{User engagement}

The RESULTs user needs study (Beetham, 2001a) suggested that on visiting the portal, academic teaching staff will have different needs from intermediaries such as educational developers, learning technologists, IT developers, librarians, managers, project staff or researchers. One way to view the needs of potential users is to identify questions that the various types of user are likely to ask. Three original areas of interest for developing RESULTs were addressed in the study. Key findings are presented here for each area.

\section{Developing artefacts for sharing practice knowledge}

Artefacts that are most effective in helping to transfer practice knowledge from one context to another were explored with a view to identifying resource types and availability of these within the community.

The user study distinguished resources that informed practice from those that were most likely to be adopted in practice. The gap between encountering an idea and making practical use of it was smallest in the case of interactive resources, such as frameworks, 
guidelines and tools (Beetham, 2001b). While academic staff preferred materials with an exact fit to their own needs, intermediaries wanted adaptable resources. In theory, many of the stated requirements could be met by materials currently used in practice, re-purposed for local support needs, such as guidance notes and workshops. This was precisely the approach taken by a ScotCIT project, SeSDL (http://www.sesdl.scotcit.ac.uk), in developing a Web-based resource centre to encourage sharing and reuse of staff development materials. In this case, it might be argued that the most effective materials are those that are most readily adaptable and usable by other staff and educational developers, rather than those which are most directly accessible for academics (Beetham, 2001b). In reality, the difficulties in terms of user participation are with regard to metadata description and the conflict of interest between user needs for rich descriptions and provider requirements, including expertise and the time to create informative metadata.

\section{Developing forums for discussion}

Forms of online dialogue that can support effective sharing of practice and the role and integration of local (offline) dialogue were explored. Learning technology specialists are required to undertake continuous learning as their area of expertise moves on. Normal practice could be described as 'peer supported experimentation'. Collaborative online communities of practice and virtual play spaces are seen as valuable opportunities to engage in this. Academic staff also needed dialogues, but favoured face-to-face relationships in their own context of practice. All those surveyed emphasized the value of examples and experiences from practice and opportunities for communication: discussion, reflection, networking and peer learning, from their desktop, and as needed. Networking is regarded as a high priority by all staff, though given the choice they prioritize contacts, events and discussion forums over access to materials and resources.

Users emphasized the need for easy-to-use tools for engaging with the online community: finding, contributing and commenting on resources and for locating other people with relevant expertise. Good examples include the SOSIG 'grapevine' facility for contacting other people with similar interests and the Amazon intelligent search results with links to reviews. Users valued push forms of information dispersal for current information providing it could be filtered for relevance.

\section{Developing networks and communities of practice}

It was important to identify the potential users of, and contributors to, the portal and their requirements and motivations to participate. Previous user needs analyses for the Resource Discovery Network (RDN) portals have been able to focus on a specific subject community, and its need for discipline- and content-based resources in relation to a relatively well-established set of academic practices - teaching and learning. Learning technology users were likely to be found from both this set and from those in a multitude of roles across discipline and institutional communities, engaged in poorly defined, yet often innovative, practices, and requiring support that is practice- rather than content-focused. There were debates over whether a potential RESULTs community was really a fairly small inner core of learning technology specialists, or the large periphery of casual and potential learning technology users, including academic staff and educational researchers.

We explored how practitioners collaborate with one another offline and how active online participations can be encouraged and supported so that it is integrated and self-sustaining 
within an overall professional development activity. The study revealed an apparent conflict between the problem-solving culture of much learning and teaching practice - the 'instant gratification' of a tip or trick discovered from the academic desktop, or the more considered solutions arrived at by collective brainstorming and the long-term habits of reflective practice and professional scholarship. A portal that focused only on this problem-solving culture would be doing little to raise the status and scholarship of teaching.

Those surveyed felt that a national service to support communities of practice in learning technology might best focus on direct, personal participation by its core community, but work towards indirect participation (for example, via LTSN subject portals or institutional VLEs) by a much broader community of users. It is likely that many users will continue to seek peer exchange, services and benefits through offline networks, and many people are likely to turn to online support only when avenues of face-to-face help have been unproductive. The survey identified a desire for national updates via a briefing service, calendar of events, news service and integration of face-to-face workshops. A directory of expertise and various types of discussion areas were also specified.

\section{Development of the RESULTs network}

Bringing together the above elements was a key aim of RESULTs. Three main structural lists were identified for use in RESULTs: user types, resource types and interaction types (Beetham, 2001a, 2001b; but see also Goodyear and Steeples, 1998, and Oliver and Conole, 2001). Functionally, professional development networks might usefully be grouped into two broad areas: facilities for interaction with artefacts; facilities for interaction with individuals/groups; with a third hybrid area of facilities for developing or enriching artefacts (Mulholland, Domingue, Zdrahal and Hatala, 2000), which is in itself a form of indirect communication with others. For RESULTs, it was felt that these different facilities needed to be seamlessly integrated to support the full representations of practice identified. They also needed to be capable of evolution as users discover new ways of interacting with one another and with their shared resources.

The RESULTs environment comprises three areas: 'MyRESULTs', 'Resources' and 'Communities'. Visually, these appear separate on the site (Figure 1) but are functionally integrated through associations between resources, users and practice. However, the distinction serves as a reminder that RESULTs is not concerned principally with resources but with resources that relate to practice. Resource metadata is kept to a minimum taking into account the conflict between the needs of contributors and users identified in the study area concerned with developing artefacts for sharing practice knowledge. The focus of description is on their use in practice. It is intended that resources are constantly commented upon, updated, contextualized, organically developed - in fact, resources should simply be contingent traces of the community process, rather than ends in themselves.

The RESULTs portal is based on a LAMP (Linux, Apache, MySQL, PHP) architecture and demonstrates a wide range of user-orientated, practice-centred functionality and interoperable Web technologies (Richardson and Powell, 2003; Richardson, Childs and Dempster, in preparation; for more information, see the CETIS Website) and an integrated discussion board (Phortm) on which a narrative of RESULTs design and development can be found.) 


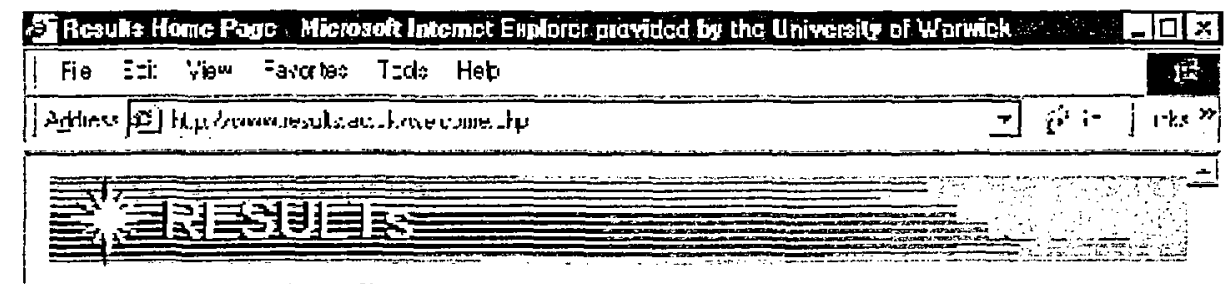

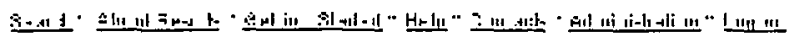

Wolcome Jay Dempitor

\begin{tabular}{|c|}
\hline MyResults: \\
\hline 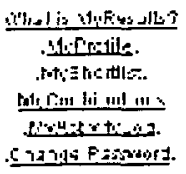 \\
\hline
\end{tabular}
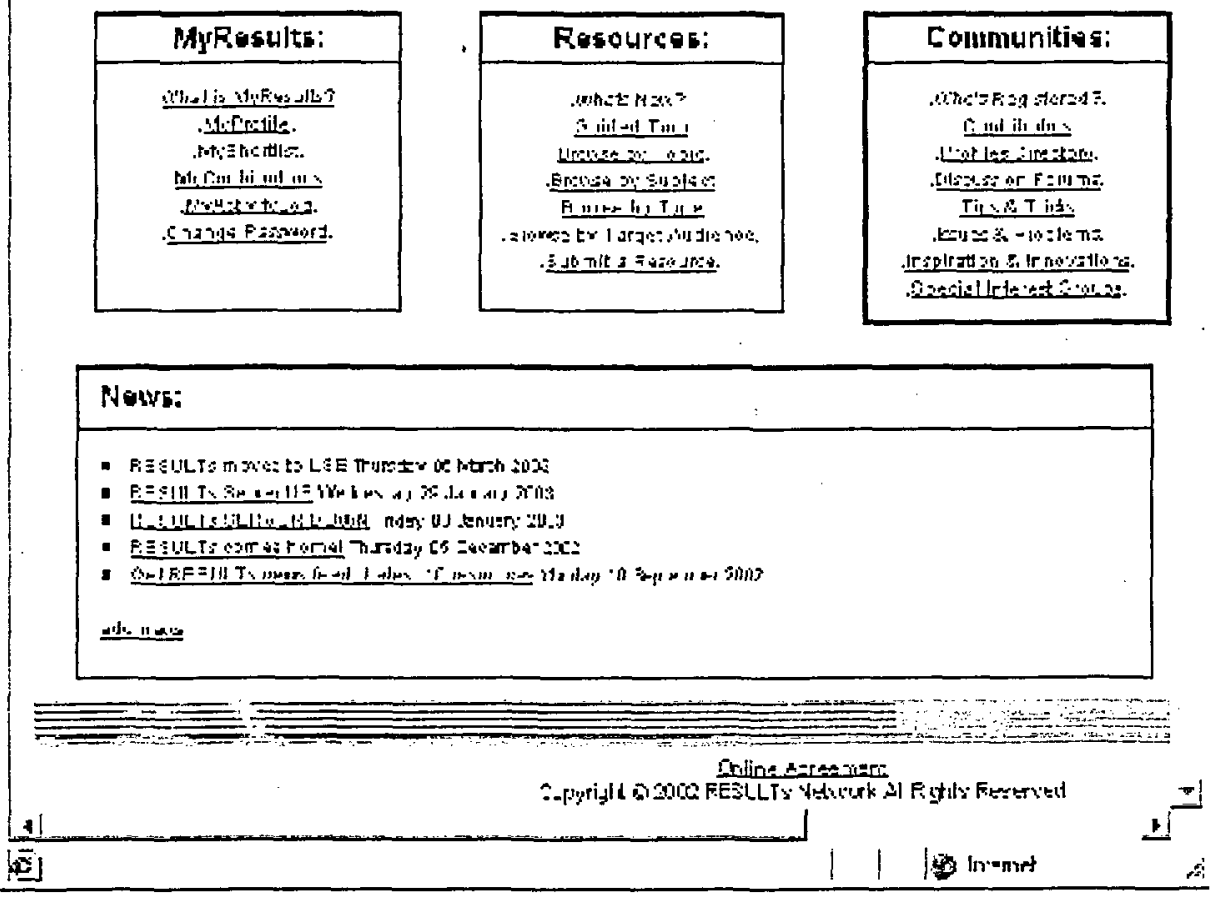

Figure 1: The RESULTS welcome page (arrived at after registration or login)

\section{User features - the visual environment}

\section{Resources and the category tree}

The category tree (Figure 2) forms only an initial taxonomy for learning technology (Browse by Topic), comprised of a 'Learning and Teaching' classification suggested by the ILTHE/LTSN (Sally Brown and Brenda Smith) and a 'Learning Technology' classification suggested by Beetham (2001a). A Guided Tour (Figure 1) provides a section of introductory materials, case studies and frameworks: written by expert practitioners for new practitioners, commissioned by national agencies or developed by research projects.

RESULTs demonstrates the integration of a number of key areas of need identified in the research study (Beetham, 2001a). Figure 3 shows the relationship between user 


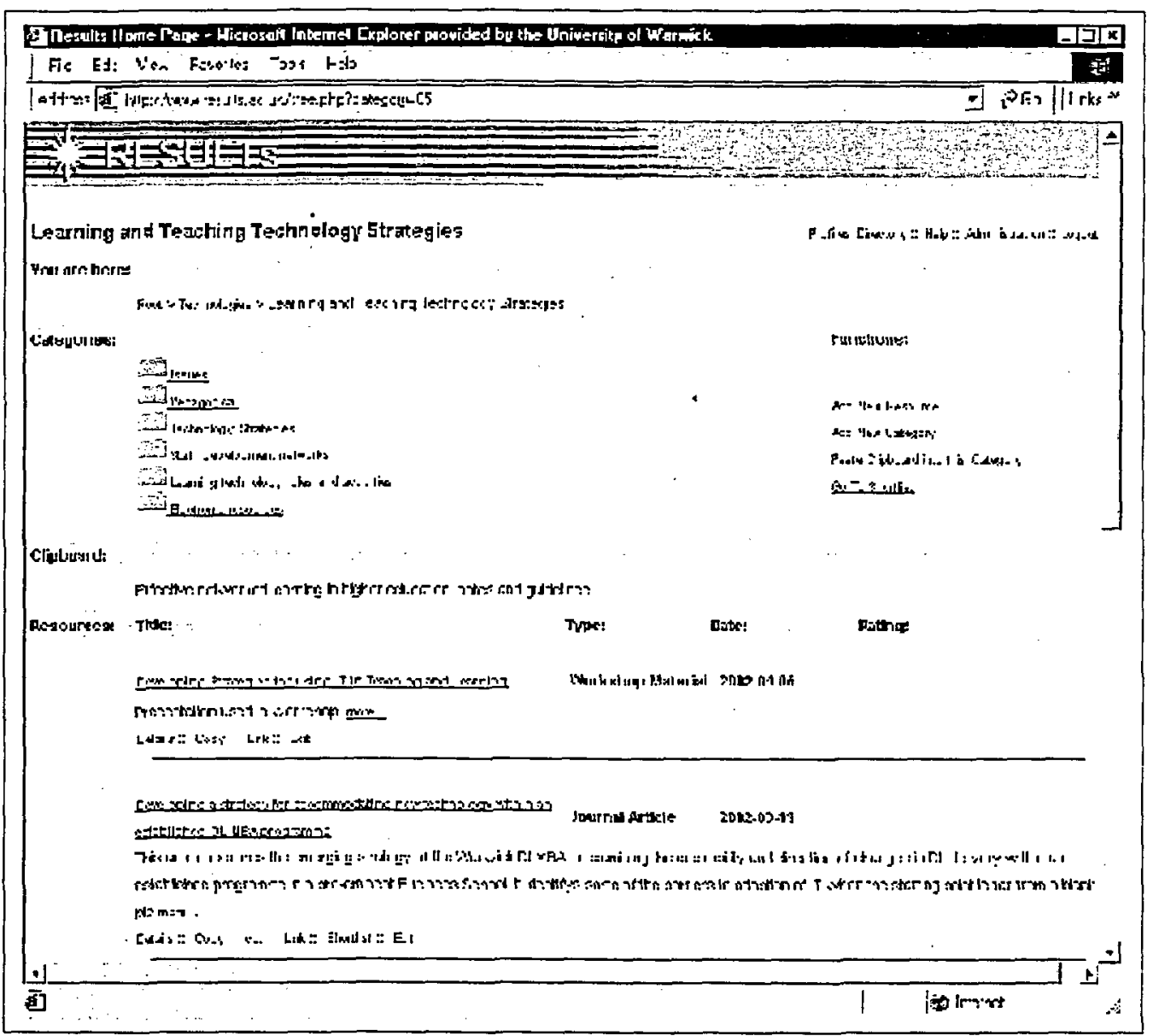

Figure 2: Browsing the RESULTS Category Tree. Top left - location in the category tree; middle left resource copied from elsewhere; top right - add new resources or categories; bottom left resource-associated functions.

requirements and design features in terms of mapping user engagement and participation activities to the online functions and facilities in RESULTs.

\section{Linking resources to people and communities of practice}

RESULTs enables meaningful associations to be made by the users between resources as they relate to practice and to their own interests through facilities to:

- Copy/cut/paste resources around the category tree;

- Link resources (including that linked resources appear immediately after linking);

- Shortlist resources to a personal collection in the user's MyResults area; and

- Start discussions from resources (function available from the Details of the resource record). 


\begin{tabular}{|c|c|c|c|c|}
\hline . & REQUIREMENTS & ACTIVITIES & FUNCTIONS & FACILITIES \\
\hline PRESENTATION & request resources & $\begin{array}{l}\text { browse, } \\
\text { search }\end{array}$ & $\begin{array}{l}\text { Browse by topic, } \\
\text { List by subject, type, } \\
\text { Target audience }\end{array}$ & $\begin{array}{l}\text { Category tree } \\
\text { MyContributions } \\
\text { (MyPreferences) }\end{array}$ \\
\hline COLLECTION & $\begin{array}{l}\text { create categories, } \\
\text { move resources, } \\
\text { collate resources }\end{array}$ & shortlist & $\begin{array}{l}\text { Add new category, } \\
\text { Shortlist }\end{array}$ & MyShortlist \\
\hline INTERACTION & $\begin{array}{l}\text { use resources } \\
\text { or interact with } \\
\text { others }\end{array}$ & $\begin{array}{l}\text { analyse, } \\
\text { evaluate, } \\
\text { discuss }\end{array}$ & $\begin{array}{l}\text { Comments metadata } \\
\text { field; } \\
\text { Copy/cut/Paste } \\
\text { Edit; Link } \\
\text { Profiles Directory }\end{array}$ & $\begin{array}{l}\text { MyProfile } \\
\text { (MyActivityLog) }\end{array}$ \\
\hline PRODUCTION & $\begin{array}{l}\text { author and develop } \\
\text { new resources }\end{array}$ & $\begin{array}{l}\text { synthesise } \\
\text { editorials }\end{array}$ & $\begin{array}{l}\text { Add new resource } \\
\text { Commissioned }\end{array}$ & $\begin{array}{l}\text { MyContributions } \\
\text { Guided Tour }\end{array}$ \\
\hline COMMUNICATION & $\begin{array}{l}\text { submit resources and } \\
\text { discuss practice } \\
\end{array}$ & $\begin{array}{l}\text { disseminate, } \\
\text { share, review, } \\
\text { debate }\end{array}$ & $\begin{array}{l}\text { Edit profile, } \\
\text { Start discussion; } \\
\text { (Rate/Review/ } \\
\text { Forward) }\end{array}$ & $\begin{array}{l}\text { MyProfile } \\
\text { Discussion } \\
\text { Forums } \\
\text { Tips, Issues, } \\
\text { Innovations } \\
\text { Special Interest } \\
\text { Groups }\end{array}$ \\
\hline
\end{tabular}

Figure 3: Passive to active engagement within RESULTs (functions/facilities shown in brackets were those that could not be implemented within the project timescale, but which the system can support straightfonwardy)

Personal development profiling is an emergent feature. From the MyResults area, users develop a personal profile of expertise ( $M y$ Profile: initially created on registration) and can list their own contributions ( $M y$ Contributions). The system can also support production of a record of activities on RESULTs (MyActivity Log).

Under Communities (Figure 1), a 'directory of expertise' is available in the collective Profiles Directory providing a source of contacts for finding expertise or common areas of interest. Users can also share approaches in practice: by contributing to Tips and Tricks, Issues and Problems, Inspirations and Innovations. They can start or contribute to Discussion Forums: associated with a resource or independently. Special interest groups are available for sharing resources amongst specific collaborative groupings.

\section{Technical developments - behind the scenes}

Metadata submission and forms handling

Although user demands for searchable and well-contextualized resources suggest the use of standard templates for submission of metadata, this is not a straightforward task. First, 
the Learning Object Metadata standard does not extend to describing resources for which the primary intended user is not the learner. Second, potential end-users had concerns about the time involved in submitting metadata. RESULTs developed a form handling application and a lightweight approach to resource description to cope with the demands of submission process and user interaction.

\section{Exporting taxonomy using IMS Metadata and the OAI-PMH}

RESULTs operates as a fully functional OAI-PMH (Open Archives Initiative Protocol for Metadata Harvesting) repository which means that any compliant OAI harvester can retrieve metadata records in XML format from the RESULTs database. These records are available as minimal Dublin Core or as extensive IMS records. Instructions on how to use the protocol may be found on the Open Archives Website (http://www.openarchives.org).

The categorization route(s) of a resource - may be extracted as taxonpath elements (IMS Metadata 9.2). This provides a mechanism by which resources can be programmatically imported from one database into another (via OAI-PMH) and automatically placed in the right location in the category or directory structure of the importing database system creating new categories as required should taxons (categories) from a taxonpath not exist. In this way, we can merge taxonomies very easily, starting with an empty or existing structure (Richardson et al,, in preparation). Bearing in mind that taxonomies (even established ones) are continually evolving, this is crucial.

\section{RSS feeds}

The ten most recently submitted RESULTs resources are available over an RSS feed (http://www.results.ac.ukltop10_rss.php). This is demonstrated on the LTSN Materials Education Website (http://www.materials.ac.uklfeatures/resultsproject.asp). An innovative use of RSS provides basic remote search capabilities to RESULTs (http://www. materials.ac.uk/technicallresults/search.asp). A description of the RSS service is available on the RESULTs development website (http://wrw. results.ac.uk/aar/presentations.php).

\section{z39.50}

RESULTS also runs a 339.50 server connected directly to the relational database which can return Dublin Core XML records. This server provides Boolean/multi-field search functionality for RESULTs and given more time and resources it could be opened to the world in general as a full z39.50 service. At present access to the server directly is blocked by a firewall.

\section{Key achievements and experiences}

In addressing the needs of users identified within the learning technology communities of practice, there have been two major successes in creating the RESULTs Network. The first is in the selection and development of the technologies that drive it. The second is in the proof of concept. However, RESULTs has been less successful in securing a critical mass of user participation. This is most likely to do with the need for integration and continuity within existing services offering regular face-to-face activities and off-line networks. This is discussed here as a third cultural and political issue for user participation.

\section{Technologies}

In terms of the technologies, significant success rested on development work in new and untested territories. The technologies selected as fit-for-purpose and implemented within 
the RESULTs architecture are novel (at least to higher education and the education sectors). It was necessary to resolve technical as well conceptual problems in clean and uncluttered ways and, given the relatively small nature of the RESULTs project, to do so with a single Web developer. RESULTs uses open source technologies and is not based on any proprietary software or code. It means that we have been able to draw upon and contribute to a large and developing body of knowledge and expertise.

While database-driven Websites and portals are far from new, we believe that RESULTs has been constructed and managed, from a technical point of view, in quite unique ways. All resources are submitted and managed online through the actual portal itself, and not through some intermediary content management system or human moderator. We know of no other examples of such an approach in portal systems and it works exceptionally well.

Interoperability has also been a high priority. RESULTs has gone a very long way and now represents a full complement of protocols: OAI-PMH (Protocol for Metadata Harvesting), RSS feeds and z39.50 searching (Richardson and Powell, 2003). Resource records are all Dublin Core and IMS conformant and can be exchanged using the corresponding XML schemas. We believe this represents the forefront of thinking and development in this area in the education sector.

Finally RESULTs is fast. Code has been specifically developed and rebuilt and graphical images kept to a minimum to enable the RESULTs portal to work at lightning speeds. It loads quickly, even on a machine with a standard modem connection; navigation around the portal and submissions to the portal are to all intents and purposes instantaneous.

\section{Proof of concept}

The second major success of RESULTs has been in the 'proof of concept'. The concept focused on how a dynamic, database-driven portal - one that has a clear target audience and has undertaken specific user needs analysis - can work. We have successfully linked resources and resource submission to people, working on the key metaphor of 'communities of practice'. RESULTs offers a portal that is largely owned and designed by its users. It is community driven and has the significant potential to reflect, support and integrate a complex group of user communities.

One implementation approach that was perhaps rather brave is the unmoderated resource submission. We trust our users in their professional conduct as it is open to misuse, even abuse. To our knowledge no other educational portal does this. All other services are moderated in some way, by some form of editorial or other quality control process. Indeed, the approach supports the concept of reflecting on one's own professional practices, as it requires users to exercise a high degree of reflection and judgement on the purpose and value of the resource to practice. Importantly, it also avoids resources being dumped into the database just to populate it with all and sundry.

The RESULTs environment supports learning technology communities of practice by offering an organic means for development of a taxonomy supporting the diverse subcommunities of learning technology practice to become transparent with consensus reached over a period of time. A substantial step in terms of interoperability of this concept was taken by making resources, their 'taxonpath', and the tree 'transportable', allowing embedding within other compatible databases. 


\section{User participation}

Given the short time scale of the project, the usage of the RESULTs portal site is currently modest and a critical mass is far from being reached. The reasons for the slow progression of uptake are complex and overlapping but include the innovation of user-driven and technical approaches and the pervasive 'not invented here' culture of higher education. This could have been overcome had a national agency driven and marketed it. It seems unlikely that the permanent revolution in individual and collective practice which is demanded by educational technology will ever be sustained without the support of local, face-to-face dialogues such as those offered by the more permanent national services and support bodies. Despite early measures to ensure that such partnerships were forged, particularly with the LTSN and ALT who were partners in the project from the outset, various political developments delayed any definitive commitment to host and further develop or drive the RESULTs Network. A scoping study undertaken by RESULTs (Jackson, 2002) with additional funding from the JISC focused upon identifying key issues and requirements in the development of a learning and teaching portal as a joint venture between the LTSN Generic Centre and the JISC. The study draws heavily on the conceptual framework for RESULTs, the user participation findings, technical developments and project experiences and in so doing is a major legacy for RESULTs in shaping this and other future portal developments. Through collaboration with ANGEL (Authenticated Networked Guided Environment for Learning), a JISC 5/99 DNER project at the LSE concerned with creating middleware services (http:// ANGEL www.angel.ac.uk), RESULTs is currently hosted by LSE.

\section{Conclusions and future directions}

There is still much vagueness concerning the nature of portals. It is not yet understood how people make use of portals, or whether personalization is useful. Furthermore, there needs to be a greater clarity in the relationship between national and institutional portals or between information-based portals and learning-based portals (EDNER, 2002).

What is clear from both the user participation survey and our own experiences, at least for professional development networks, is the need to build links between virtual 'portal' activities and face-to-face activities such as workshops and conferences. The portal must endeavour to become a part of the wider communities and networks - which means the portal must become the online part of the communities and networks that users already belong to. The notion of roving facilitators to bridge online and offline communities of practice is one possible mechanism for managing continual engagement of practitioners. Directed and scaffolded support is likely to motivate participation in both online and offline professional development, such as that provided through the structured and formal framework of activities and opportunities within CPD accredited programmes. (Indeed, SEDA, ALT and the ILTHE are exploring routes specifically for learning technologists.) As the portal is seen to offer facilities that are valuable, timely, engaging, fruitful and inspiring, word will consequently pass around those networks, and users may then start to turn to the portal as a first priority.

There is a strong indication that RESULTs will be sustainable in the long term only as part of more general learning and teaching portals and only providing that it is cross-searchable from the subject portals which are becoming the recognized repositories of practice-based 
information for United Kingdom higher education. Integration of learning technology communities of practice would thus incorporate users who may utilize more specific or local entry points. There is a danger that a focus on access to information and resources per $s e$ will allow portals to become perceived as administrative repositories simply affording access to the 'knowledge' that the user needs, rather than offering tools for educational annotation and thus supporting shared professional scholarship and growth.

On balance, RESULTs has been successful in developing a portal system that offers a valuable range of professional exchange functionality and interoperability. Given the collective accomplishments within learning technology communities in United Kingdom higher education and further education, the only constraining factors remain with the political dimension of joined-up thinking in the aforementioned portal developments. Effective collaboration between national agencies is a key requirement, coupled with the need for sustainable development in project-initiated services: a matter for the funding councils.

\section{Acknowledgements}

RESULTs has been a worthwhile project and we are grateful to the JISC for its initial funding and on-going support and enthusiasm for the work.

The authors wish to thank the wide range of learning technologists, educational and staff developers, academic staff and many other researchers and practitioners for their involvement and commitment to exploring the conceptual and technical parameters on which portal development relies. The journey towards providing a one-for-all, lively and effective online community of practice for learning technology has really only just begun.

\section{References}

Armitage, S., Rothery, J. and Jenkins, M. (1999), 'Report of a UCISA survey of support provided in universities and colleges for the use of technology in teaching and learning', Universities and Colleges Information Systems Association: Teaching, Learning and Information Group. Also available on the Web: http://www.ucisa.ac.uk/TLIG/teach/docs/ survey991

ASTER Project (1999), A survey of current practice in the use of C and IT to support small-group teaching activities in disciplines associated with the Humanities, Physics and Psychology', accessed on the Web 5 March 2003, http://cti-psy.york.ac.uk/aster/resources/ publications/Report_2/report2.pdf

Baecker, R. (1992), Readings in Computer-supported Co-operative Work, Morgan Kaufmann Publishers, Inc.

Beaty, E. and McGill, I. (1995), Action Learning: A Guide for Professional, Management and Educational Development, London: Kogan Page.

Beetham, H. (2000), 'Learning technology scoping study: lessons for educational developers', Educational Developments, 1, 4.

Beetham, H. (2001a), 'User needs study for the RESULTs project', accessed on the Web 28 February 2003, http://www.results.ac.uk/aar/documents/user_needs.doc 
Beetham, H. (2001b), 'How do representations of our practice enable change to happen?', Educational Developments, 2, 4, 19-22, accessed on the Web 7 March 2003: http://www.eres. ac. $u k /$ sourceldocs/pub-ou-47.pdf

Beetham, H. (2002), 'Developing learning technology networks through shared representations of practice', in C. Rust (ed.), Improving Student Learning Through Learning Technologies, Oxford: Centre for Staff and Learning Development, accessed on the Web 7 March 2003, http://www.eres.ac.uk/source/docs/pub-ou-53.pdf

Beetham, H., Jones, S. and Gornall, L. (2001); Career Development of Learning Technology Staff: Scoping Study Final Report, accessed on the Web 3 March 2003: http://sh.plym.ac.ukledsleffects/jcalt-project/final_report_v8.doc

CETIS, Centre for Educational Technology Interoperability Standards, http://www.cetis.ac.uk/ Conole, G. C. (2003), 'The evolving landscape of learning technology', $A L T-J, 10$ (3), 4-18.

EDNER (Formative Evaluation of the Distributed National Electronic Resource) Project (2002), Portals in Higher and Further Education, Issues Paper 5, accessed on the Web: 27 March 2003, http:/lwww.cerlim.ac.ukledner/iplip05.rtf

Foster, J., Bowskill, N., Lally, V. and McConnell, D. (2000), 'Researching community: developing a design for a virtual professional development center in computer based collaborative group work', paper presented to International Consortium for Educational Development in Higher Education, 22-6 July, Bielefeld, Germany.

Gibbs, G. (2000), 'Learning and teaching strategies: the implications for educational development', Educational Development, 1.1 (1), 3-5,

http://www.seda.ac.ukled_devs/voll/Eddevs11.htm

Goodyear, P. and Steeples, C. (1998), 'Creating shareable representations of practice', ALT-J, 6 (3), 16-23.

Gornall, L. (1999) 'New professionals: change and occupational roles in higher education', in Perspectives: Policy and Practice in Higher Education, 3 (2), 44-9.

Jackson, P. (2002), 'Scoping study for a learning and teaching portal for HE', unpublished report to the JISC/LTSN.

Kollock, P. and Smith, M. (2000), 'Communities in cyberspace', in M. Smith and P. Kollocks (eds), Communities in Cyberspace, London: Routledge.

Lally, V. and McConnell, D. (undated), 'Networked professional development: a review of current practice', Institute of Education Research Topic Papers, University of Sheffield, accessed on the Web 3 October 2003 at, http://www.shef.ac.ukleducation/researchl RTPLandMc.shtml

Lave, J. and Wenger, E. (1991), Situated Learning: Legitimate, Peripheral Participation, Cambridge: Cambridge University Press.

McConnell, D. (2000), Implementing Computer Supported Cooperative Learning (2nd edn), London: Kogan Page.

Mulholland, P., Domingue, J., Zdrahal, Z. and Hatala, M. (2000), 'Supporting 
organisational learning: an overview of the ENRICH approach', Journal of Information Services and Use, 20, 1, 9-23, accessed on the Web 7 March 2003 at, http:/lkmi.open. ac.uklprojects/enrich/ISU-Enrich.pdf

National Committee of Inquiry into Higher Education (NCIHE) (1997), Higher Education in the Learning Society, Bristol, Higher Education Funding Council for England, accessed on the Web 7 March 2003 at, http://www.leeds.ac.ukleducol/ncihel

Oliver, M. (2002), 'What do learning technologists do?', Innovations in Education and Teaching International, 39, 4, 1-8.

Oliver, M. and Conole, G. C. (2001), 'Supporting structured change: toolkits for design and evaluation', Chapter 6 in R. Macdonald (ed.), Academic and Educational Development: Research, Evaluation and Changing Practice in Higher Education, SEDA Research Series, London: Kogan Page, 62-75.

Richardson, S., Childs, M. and Dempster, J. A. (in preparation), 'Developing interoperable taxonomies for sharing resources within multidisciplinary communities of practice', submitted October 2003 to International Journal of Interactive Technology and Smart Education (new journal: http://ww'w troubador.co.uk/itse/)

Richardson, S. and Powell, A. (2003), 'Exposing information resources for e-learning harvesting and searching IMS metadata using the OAI Protocol for metadata harvesting and Z39.50 Ariadne 34', accessed on the Web 28 February 2003 at www.ariadne.ac.ukl issue34lpowell!

Stefik, M. (1995), Introduction to Knowledge Systems, Morgan Kaufmann Publishers.

TALiSMAN (1999), 'Review of staff development courses and materials for C and IT in teaching, learning and assessment', http://www:talisman.hw:ac.ukl 